\title{
Comparative Analysis of Wear Resistance of Non-Smooth Metal Surface between Laser and Machining
}

\author{
Zhuojuan Yang ${ }^{1, a}$, Youquan Chen ${ }^{2, b}$, Lidan Fan ${ }^{1, c^{*}}$ \\ 1College of Mechanical Engineering Jilin Teachers' Institute of Engineering \& Technology \\ Changchun, China \\ ${ }^{2}$ Department of Mechanical Engineering Changchun Institute of Engineering Technology \\ Changchun, China \\ ayangfeng@163.com, ㅁouan3168@sina.com, ${ }^{c} 574024123 @ q q . c o m *$
}

Keywords: Non-smooth metal surface; Laser; Wear resistance; Non smooth wearable technology;

\begin{abstract}
According to the biological non smooth theory, the design of non smooth surface morphology, mechanical and laser processing method, the formation of similar organisms form non smooth surface on the surface of 45 steel; wear test, laser machining and smooth specimen samples and samples in the bionic micro friction wear test machine, the test results of qualitative and quantitative analysis non smooth surface in the mechanical parts wear resistance of the role; the smooth, bionic coupling, mechanical processing of wear test, found that wear resistance of specimens with optimal laser bionic coupling surface, followed by machining the bionic sample, unprocessed smooth surface wear resistance of the sample is the worst.
\end{abstract}

\section{Introduction}

Where there is mechanical movement there is friction and wear problems exist, which is bound to be accompanied by energy consumption, material losses, as well as the replacement of parts and repair caused by the waste of human resources. Wear is one of the three main forms of failure of metal mechanical parts (wear, fatigue, corrosion). According to incomplete statistics, the world's energy $1 / 3$ to $1 / 2$ consumed in wear [1]. Statistics of 500 typical parts of foreign countries show that, due to wear and tear caused by about $80 \%$ [2], so the wear problem is one of the important basic scientific problems in the mechanical industry.

The traditional concept that tribological parts surface more smooth more anti friction [3], but domestic and foreign scholars in recent 30 years, the results of the study show that [4] has a certain degree of corrugated surface parts is easy to store lubricating oil, the oil film formed on the surface of parts resistance to continuous severe wear, but the surface is too smooth, due to lack of waviness the formation of oil film, anti bonding capacity is very low, but due to the friction caused by the metal surface of the asperity contact and adhesion, reduce the wear resistance. For this purpose, the scholars of various countries have carried out a series of studies in this area.

\section{Development Status and Trends at Home and Abroad}

Abroad proposed the non smooth wearable technology [5] in 1980s, Rohde S M was first proposed in 1980 by the micro - Method of reducing friction smooth surface; surface pits of different sizes to simulate the piston ring and cylinder system by Ronen $\mathrm{A}[6]$ et al., the results show that the average friction average friction surface non smooth specimens than on the smooth surface of specimens is reduced by $30 \%$ or more.

Non smooth sealing surface pits Etsion [7]1997 on several different processing by laser were studied. The results show that the best non smooth surface morphology is spherical pit and the existence of the best depth of the oil film stiffness of the largest diameter ratio. In1999 and other years in the test chamber of the pump in the pump seal test Etsion study shows that the smooth sealing surface can be seen wear traces, rather than the smooth surface of the slightest [8]. Therefore, it is one of the 
developing directions of the mechanical seal in the future to carry out the appropriate non smooth processing of the sealing surface.

In order to improve the wear resistance of cold roller, in recent years the special modulation of the work roll surface with high repetition rate, high energy density, high power pulsed YAG laser beam on the surface of the roller, have non smooth surface to improve its wear resistance. The application shows that the use effect and service life of the cold rolling mill are obviously improved after laser treatment. In the 20 roll of Sendzimir Mill with the cold rolled low carbon steel plate, the roller life more than 3 times than the ordinary roller; with its flat annealed low carbon steel plate, the service life can be compared with the common roll increased more than 5 times, even more than 10 times [9]. In the ordinary two roller mill, with the bionic coupling roller cold rolling high strength $65 \mathrm{Mn}$ spring steel, the service life is 2 3 times of the ordinary roller [10].

Key Laboratory of Ministry of education Jilin University bionic engineering in recent years in Ren Luquan under the leadership of academician in the biological non smooth desorption, reducing resistance and wear and do a lot of research work, made a series of research results.

Academician Ren Luquan et al studied pangolin, snakes and lizards and other reptiles, reptiles found in a sand body, has remained intact and was never injury, skin friction and wear behavior of pangolin scales were investigated, found in a period of time after the friction test and reach steady state after wear, wear the obvious non smooth the surface morphology of presentation, showed very good wear resistance. At the same time, it is found that the surface of soil animal is not only in the form of non smooth characteristics, but also the body surface of the cell body and the mother of different material composition and non smooth structure. According to the biological surface wear resistance effect of non smooth morphology and structure, applied to the relative motion of mechanical components (slider, drill pipe, roll surface, etc.) can significantly improve the wear resistance of the relative motion of mechanical parts surface, friction resistance and adhesion force and relative motion of small. Since then, the bionic non smooth surface has been widely used in the mechanical wear parts. When compared with the surface of the cylinder and the piston, the cylinder liner and piston wear resistance of the cylinder liner and piston were found to have more than 4 times as many as the smooth surface. Non smooth wear resistant principle is applied to hot rolling mill roll for rolling low carbon steel plate, the roller life increased by more than 3 times. The development trend of non-smooth wear resistant technology is as follows.

(1) The mathematical model of the non smooth surface and the dynamic expression of the computer. For some typical aquatic and terrestrial plants and animals and the environment effect of wear surface, on the basis of different exercise style and classification, selection of basic types of mathematical expression, so as to realize the expression of biological surface computer by characteristic parameters of mathematical surface control.

(2) Quantitative analysis and qualitative analysis of the wear mechanism and the influence of surface morphology parameters on the wear resistance of typical biological wear resistant surface.

(3) To improve the manufacturing technology of bionic wear-resistant surface, expand the scope of application of bionic non smooth wearable technology.

\section{Laser Processing and Mechanical Processing of Samples of Wear and Tear Test Research}

In summary, in recent years the technology of non smooth wear resistance is mainly focused on the study of the wear resistance of laser processing non smooth surface. The laser because of its high strength, high density and the wear resistance is improved to strengthen the role of the material itself, it has not yet been effect of smooth surface morphology and density distribution parameters on the wear resistance of the contribution rate. To this end, the project intends to carry out laser processing and mechanical processing of samples of wear and tear test research, the research significance of the project is mainly reflected in the following two aspects:

(1) The effect of the surface topography on the wear resistance of non smooth surface is quantitatively explained by the contrast test of the wear of the laser machining and the machining; 
(2) Through the wear test machining samples, optimized test range of non smooth surface morphology, size and distribution, the experimental study and theoretical analysis results will provide new ideas and design basis for the design of non smooth surface friction.

\section{Main Research Contents}

According to the biological non smooth theory, the design of non smooth surface morphology, mechanical and laser processing method, the formation of similar organisms form non smooth surface on the surface of 45 steel; wear test, laser machining and smooth specimen samples and samples in the bionic micro friction wear test machine, the test results of qualitative and quantitative analysis non smooth surface in the mechanical parts wear resistance in action; optimized test within the scope of the best wear morphology, size and distribution of non smooth surface; wear mechanism analysis is the main research contents of this project, including the following:

1. Non-smooth shape, size and distribution density of the design, laser and mechanical processing of sample preparation

(1) The non smooth shape, size and distribution density are selected by the analysis of the surface features of the biological wear resistance.

(2) Laser and mechanical processing methods of different shapes, different sizes and different distribution densities.

2. Wear test of specimens

(1) The contrast test of the smooth, the same morphology of laser and mechanical processing samples. The non smooth wearable effect explanation mainly in the following two aspects: one is the laser processing in the surface enhanced due to the improvement of wear resistance, this is the wear resistance of a part to improve the surface modification; two is a non smooth surface effect. Through this part of the test, wear resistance effect is smooth and uniform morphology and laser machining of the sample, study on the wear resistance of laser strengthening contribution rate, to further explore the non smooth unit shape, size and distribution of the wear resistance of the role.

(2) Wear testing of non smooth surface specimens with different machining processes.

First, the wear samples of different non smooth specimens were designed to determine the best shape. After that, the shape of different size, different distribution and different distribution of the samples were not smooth.

3. Wear mechanism of non smooth topography

The experimental design optimization theory to establish the size, distribution and other factors regression model between wear volume, based on the experimental results and theoretical analysis, to explore the relationship between the non smooth wear resistance and unit size, distribution density and other factors, and reveal its mechanism and wear.

\section{Steel Friction and Wear test}

Test specimens of the substrate material is 45\# steel, Quenched and tempered, will be large 45\# steel shaped steel wire cut into the required size of the test, that is, $20 \mathrm{~mm} \times 10 \mathrm{~mm} \times 8 \mathrm{~mm}$. From the test plan Table 4-2, we can know that the test process, there are 28 kinds of test specimens, the 84 test, in order to reduce the impact of the test error, each test number were done 3 times. Test steps are as follows:

1.In order to keep the specimen surface clean, in order to reduce the experimental error, with ethanol before the test in medical ultrasonic cleaner cleaning in 20 minutes, and then in the water for 10 minutes with ultrasonic cleaning, hair dryer, in an electronic balance weighing down as before - wear weight;

2.It is fixed on the sample table of the micro tribology experiment machine. The GCr15 steel ball with a diameter of 63 and HRC 4, as the grinding element, was chosen as the grinding element. On the test piece to apply $10 \mathrm{KG}$ force on the grinding, each time along the friction surface to walk 3 times, about 90 minutes;

3. Anhydrous ethanol in medical ultrasonic cleaner cleaning in 20 minutes, and then in the water for 10 minutes with ultrasonic cleaning, hair dryer, weighing as - wear weight in electronic balance; 
4.Samples in the direction perpendicular to the moving direction of the laser longitudinally, embedded and made of grinding and polishing of metallographic samples with $4 \%$ nitric acid alcohol solution corrosion, using Japanese JSM-5310 scanning electron microscope (Scanning Electron microscope observation samples, the test voltage is $25 \mathrm{kV}$, the magnification is 5000 times.

\section{Bionic Coupling Sample and Mechanical Processing Sample}

The geometrical morphology of machining specimen is concave, the diameter of $d=1 \mathrm{~mm}$, the center distance were $a=1.5 \mathrm{~mm}, a=2 \mathrm{~mm}$ and $\mathrm{a}=2.5 \mathrm{~mm}$, three samples, the same Laser Biomimetic Coupling samples geometric shape and size of the wear weight loss comparison curve, as shown in Fig.1.

As can be seen from Fig.1, under the same micro geometry conditions, the laser bionic coupling sample is less than mechanical processing, which shows that the laser bionic coupling surface is more resistant to wear.

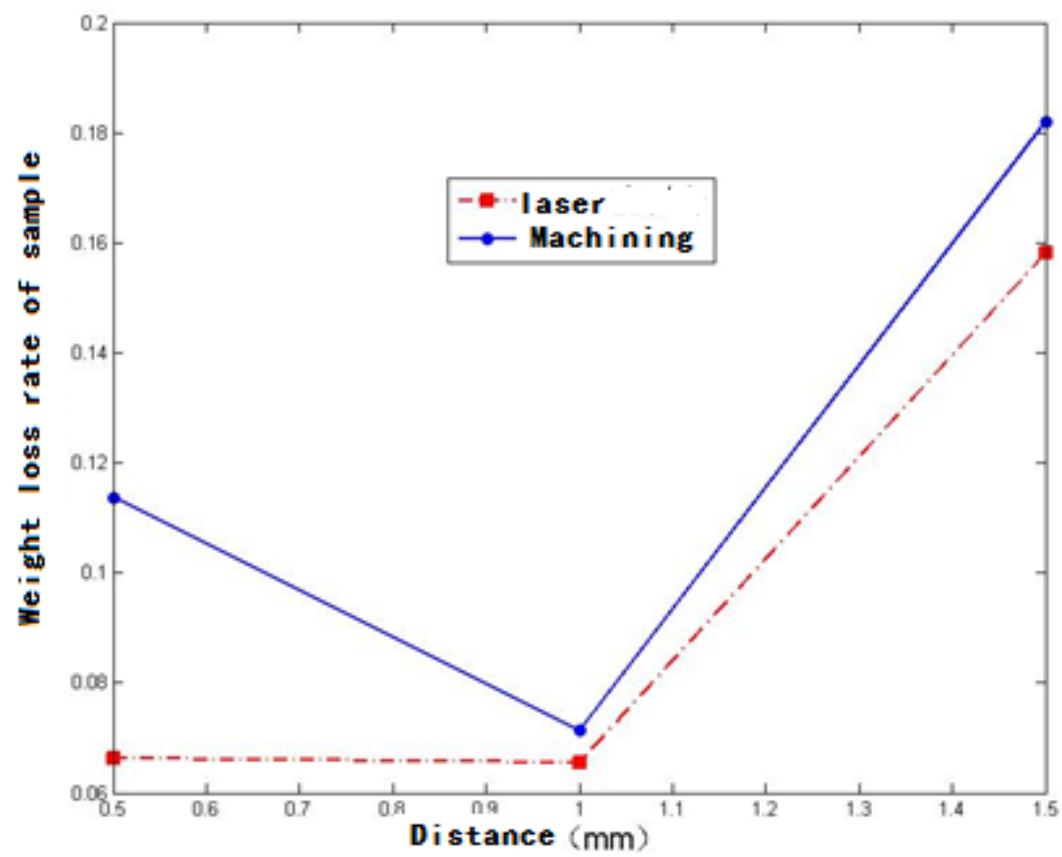

Figure 1. Comparison of the loss of weight after the laser bionic coupling and the mechanical processing sample

\section{References}

[1] Yuri Kligerman, Izhak Etsion. Analysis of the hydrodynamic effects in a surface textured circumferential gas seal. Tribology Transactions, 2001,44(3): 472-478

[2] Jiho Uh, Jin Lee, Yoon H. Kim, et.al. Laser engraving of micro-patterns on roll surfaces, ISIJ International, 2002, 42(11): 1266-1272

[3] A Skopp, M Woydt and K H Habig. Unlubricated sliding friction and wear of various Si3N4 pairs between 22 10000C. Tribology International, 1980, 12 (3): 153-158.

[4] Nakada M. Trends in engine technology and tribology. Trib.Int' 1.,1994, (27): 3-8

[5] Ronen A, Etsion I, Kligerman Y. Friction-reducing surface-texturing in reciprocating automotive component, Trib.Trans., 2001,(44): 359-366

[6] Ryk G, Ligerman Y K, Etsion I. Experimental investigation of laser surface texturing for reciprocating automotive components. Tri. Trans. 2002 , 45(4): 444-449 
[7] Etision I, Burstein L. A model for mechanical seals with regular microsurface structure. Trib.Trans.,1996,39(3):677-683

[8] Etsion I, Halperin G. Greenberg Y. Increasing mechanical seal life with laser-textured seal faces. In Proc.of 15th Int'1.Conf. Maastricht. 1997:3-11

[9] Etsion I, Kligerman Y. Analytical and experimental investigation of laser-textured mechanical seal faces, Trib. Trans., 1999,42(3): 511-516

[10] Ujihara S. Iron and Steel Engineer, August, 1991,52:163-16. 